\title{
A Comprehensive Parametric Analysis of Vertical Handoff in Next Generation Wireless Networks
}

\author{
${ }^{1}$ Mrs.Jayasheela C S, ${ }^{2}$ Dr. Gowrishankar \\ ${ }^{I}$ Dept. of ISE, BIT, Bangalore, India, \\ ${ }^{2} H O D$, Dept. of CSE, BMSCE, Bangalore, India
}

\begin{abstract}
The science and technology is developing day by day leading to expand and forcing to reshape our living standards. The main aspects leading to deployment of a wide array of wireless and mobile networks are advances in miniaturization, low power circuit design and development in radio access technologies and increase in user demand for high speed internet access. The varying wireless technologies are driving today's wireless networks to become heterogeneous, ie., the network comprised of two or more different access network technologies. Heterogeneous networks have created many challenges such as mobility management, handoff, resource management, location management, providing QoS, security and pricing. Switching between heterogeneous networks requires seamless vertical handoff. The selection of parameters plays an important role in the decision of vertical handoff. The parameters like bandwidth, signal strength, velocity, power consumption, throughput, cost, user preferences and network load are considered during vertical handoff. In this paper, the vertical handoff parameters of the existing work are critically ascertained and presented in a tabular format for identifying the important parameters. A dynamic, adaptive and efficient approach is required to implement the vertical handoff mechanism in next generation heterogeneous wireless networks to produce an effective service for the user by considering dynamic and non dynamic parameters.
\end{abstract}

Keywords: Heterogeneous networks, parameters, handoff

\section{Introduction}

Handoff management is one of the components of mobility management which controls the change of mobile terminal's point of attachment during active communication. Handoff is the process of changing the channel connection when the call is in progress. Handoff can be divided into two different types - horizontal and vertical. The switching between points of attachment or base stations that belong to the same network is called Horizontal handoff and this is required in homogeneous networks.

\subsection{Vertical handoff}

The switching between points of attachment or base stations that belong to the different network technologies is called Vertical handoff and this is required in heterogeneous networks. The process of Vertical handoff [23] can be divided into three steps, namely system discovery, handoff decision and handoff execution. During the system discovery, mobile terminal equipped with multiple interfaces have to determine which networks can be used and what services are available in each network. During the handoff decision phase, the mobile device determines which network it should connect to. During the handoff execution phase, connections are needed to be re-routed from the existing network to the new network in a seamless manner. This requirement refers to the Always Best Connected (ABC) concept, which includes the authentication, authorization as well as the transfer of user's context information. In heterogeneous networks, the need for vertical handoff can be initiated for convenience rather than connectivity reasons.

\section{Parameters For Vertical Handoff}

The decision for vertical handoff may depend on various parameters like Bandwidth, Received Signal Strength (RSS), Signal to inference ratio (SIR), cost, latency, security, velocity, battery power, user preferences, service capacities and Quality of service (QoS). From the literature surveyed, different research scholars have given different views and techniques to achieve vertical handoff. In order to design a Vertical Handoff (VHO) mechanism for next generation wireless networks, it is essential to study the existing VHO mechanisms. The study of existing mechanisms will assist in the identification of important parameters for VHO mechanism. As of now, a few approaches for $\mathrm{VHO}$ have been found in the literature.

\subsection{Available Bandwidth}

Bandwidth is a measure of the width of a range of frequencies. It refers to the data rate supported by a network connection or interface. It measures how much data can be sent over a specific connection in a given amount of time. In order to provide seamless handoff for Qos in wireless environment, there is a need to manage bandwidth requirement of mobile node during movement. Bandwidth is generally known as the link capacity in 
a network. Higher offered bandwidth ensures lower call dropping and call blocking probabilities; hence higher throughput. Bandwidth handling should be an integral part of any of the handoff technique.

A Novel Decision Scheme for Vertical Handoff in 4G Networks is introduced [1] and two Handoff decision schemes for Heterogeneous networks are presented. In the first scheme they introduce a score function to find the best network at best time from a set of neighboring networks. Score function uses Bandwidth, RSS and Access fee as its parameters. Second scheme makes use of classic triangle problem to find the best network from a set of neighboring networks. This problem considers three parameters Bandwidth, RSS and Access fee as the three sides of a triangle. If an equilateral triangle is obtained with these parameters of a network then that network will be the best among the set of networks. The best decision model meets the individual user needs but also improve the whole system performance by reducing the unnecessary handoffs. Since the second algorithm performs handoff only if the constraints are above the threshold value. The call dropping probability is reduced and holding time is increased.

A novel framework to evaluate the VHO algorithm design impact on system resource utilization and user perceived QoS is presented [2]. This framework can be used to compare the performance of different vertical handoff algorithms. The results provide a quantitative means to evaluate the critical impact of the handoff algorithm design on satisfying the active real-time application requirements while improving the overall resource utilization.

A call flow for vertical handover procedure and a soft QoS scheme was proposed using Dynamic Programming (DP) approach for an efficient radio resource management in an environment where several different radio access networks (e.g.,WLAN and WCDMA) coexists [3]. The soft QoS scheme is compared with hard QoS scheme for optimizing resource allocation using a Greedy and DP approach during upward vertical handover. The proposed soft QoS scheme is more efficient in network utilization than existing hard QoS scheme.

A seamless and proactive vertical handoff scheme was designed [4] based on the architecture that aims to provide always the best QoS for users. Evaluation algorithms are derived to estimate the conditions of both WiMAX and WLAN networks in terms of available bandwidth and packet delay. The results obtained prove the feasibility and effectiveness of the proposed schemes.

An access network selection algorithm was presented by extending the traditional Analytic Hierarchy Process (AHP) Multiple Criteria Decision making technique that suits the QoS requirements of applications [5]. The algorithm considers the criteria that include available bandwidth, end-to-end Delay, Jitter, Packet Loss, Cost and security of the network and Wi -Fi, Wi -Max and CDMA networks as the alternatives.

The algorithm is simulated using Java and the pair-wise comparison matrices are stored in two dimensional arrays. Applications that require three types of traffic classes namely voice traffic, video traffic and multimedia traffic were considered. The matrices are checked for consistency and proven to be consistent. Composite weights of the available networks are evaluated and the network that is having the highest composite weight is selected as the target network. Since the algorithm is based on AHP, it gives both qualitative and quantitative evaluation of the alternatives which means that it determines the optimal target network and also evaluates how best the target network is suitable for a specific traffic class.

An intelligent approach for vertical handover decision was introduced [6]. A model is proposed which gathers events from link layer, network layer and transport layer and takes decision based on fuzzy rules. The model chooses different variables, i.e. Available Bandwidth, Signal Strength \& Network load. System Simulation is done using Sugeno Fuzzy Inference system and Fuzzy inference collects input values of selected parameters from event collector as crisp inputs and then evaluates them according to rules. The composed and aggregated output of rules evaluation is defuzzified and crisp output is obtained. The output of the fuzzy system is handover decision and an intelligent decision will be taken based on output values.

An UMTS-WLAN integrated architecture was proposed [7] with dynamically updating database at UMTS network which keep track of network condition such available bandwidth and designed algorithm for handoff decision when Mobile Node (MN) need to switch to other network due to poor network service. Whenever MN needs to switch to other network it takes the network condition information from database and make decision of handoff. The proposed scheme is simulated and compared with existing RSS based handoff scheme and the proposed scheme gave better performance in terms of Packet Delay Ratio(PDR), total number of handoff, total time taken for handoff, total packet loss and channel utilization.

An architecture involving an external host based light-weight server Access Link Utilization Monitor (ALUM) was proposed that disseminates the available end-to-end bandwidth to the mobile node to assist it in making a decision to maintain end to-end service quality [8]. The information it provides can be used by any VHO algorithm. A Fuzzy Logic Based Quantitative Decision Algorithm (FQDA) is presented and implemented which takes available bandwidth provided by ALUM as a decision parameter. A uniform metric conversion method is proposed to compare dissimilar (but related) parameters in the decision making. 


\subsection{Speed}

It is the speed at which the Mobile Terminal (MT) is moving. In vertical handoff algorithms, the speed factor has a large and important decisions binding effect than traditional handoff decision algorithms horizontal handoff. When the users travel at high speed within a network coverage area is discouraged the idea to initiate vertical handoff process because after a short period of time the user will have to go back to the initial network because it will get out from under cover network host.

A Fuzzy Logic-based Adaptive Handoff (FLAH) management protocol was developed [9] it was then integrated with an existing cross layer handoff protocol. The fuzzy logic based system initiates handoff using fuzzy logic; it uses mobile's speed \& distance as input and received signal strength as output. The algorithm used is adaptive to velocity interference and distances, resulting in fewer dropped calls, better communication quality, potentially lower MT transmit power requirements, give good performance at different MT speeds, and decreases handoff failure probability. Performance analysis and simulation results show that the FLAH protocol significantly enhances the performance of both intra and inter-system handoffs. It can estimate the right time to initiate handoff more precisely. It also significantly reduces the cost associated with the false handoff initiation because it achieves lower false handoff initiation probability than existing handoff protocols.

A dynamic decision model (DDMVH) to make the right vertical handoff decisions by determining the "best" network at "best" time among available networks based on, dynamic factors such as RSS of network and velocity of mobile station simultaneously with static factors like Usage expense, Link capacity (offered bandwidth) and power consumption is designed [10].

A handoff scheme (RBAHMS) which takes handoff decision adaptively based on the type of network it presently resides and it also relies on the speed of the mobile terminal to make a decision of the handoff initiation with RSS threshold value [11]. To enhance the handoff performance of mobile IP in wireless IP networks, the false handoff probability in the NGWS handoff management protocol was reduced [12]. Based on the information of false handoff probability, the effect on mobile speed and handoff signaling delay is analyzed.

The ability of Information Technology in $4 \mathrm{G}$ in Wireless Networks is demonstrated by proposing a vertical handoff scheme and discusses its operation like handoff decision making, network selection and handoff execution [13]. RSS and MN speed are the primary handoff metric. The MN speed is obtained by the GPS system and RSS information is gathered from RSS measurement function. The vertical handoff system influences the packet drop rate in tight and loose integration approach under different load conditions.

A Movement-Aware vertical (MAV) handover algorithm (MAVHWWi) between WLAN and Mobile WiMAX for seamless ubiquitous access is addressed [14]. If a Mobile Station (MS)'s velocity is high and its movement pattern is irregular, unnecessary handovers likely occur more frequently. Therefore, the MS velocity and moving pattern are important factors for the handover decision procedure. To avoid unnecessary handovers, the MAV handover algorithm adjusts the dwell time adaptively and predicts the residual time in the cell of target Base Station (BS).

A novel handover algorithm (DSVHAVC) in heterogeneous radio network for intelligent transportation system [15], a method decides the priority of radio access network that is most suitable for user's application at a particular vehicular speed in the constraint resource environment. This algorithm is specific to vehicular communication system and hence variation in network selection with vehicle speed is presented. The results show that the presented model not only realistically optimizes the best available network on the move but also avoids unnecessary handovers.

\subsection{Received Signal Strength (RSS)}

RSS is the most widely used criterion because it is easy to measure and is directly related to the service quality. Majority of existing horizontal handover algorithms use RSS as the main decision criterion, and RSS is an important criterion for VHD algorithms as well, but it is not enough for a complete decision. It is the strength of the signal received, as the RSS of the neighboring network rises above the threshold the Vertical Handoff is feasible i.e. the handoff takes place if and only if RSS of the BS or Access Point (AP) is above the threshold.

A Novel Decision Scheme for Vertical Handoff in 4G Networks, which introduce a Score function which makes use of Bandwidth, RSS and Access fee as its parameters to find out best network at best time from a set of neighboring networks[1]. The scheme also makes use of classic triangle problem to find the best network from a set of neighboring networks considering the above three parameters as the three sides of a triangle. If an equilateral triangle is obtained with these parameters of a network then that network will be the best among the set of networks.

A dynamic decision model is to make the right vertical handoff decisions by determining the "best "network at "best" time among available networks [10]. The decision to decide best network is based on static factors such as the bandwidth of each network (capacity), usage charges of each network, power consumption of each network interface and battery level of mobile device and dynamic factors are considered in handoff decisions for effective network usage. Dynamic factor like RSS can help in improving whole system 
performance; current user conditions, such as a mobile host's moving speed can eliminate certain networks that do not support mobility.

A realistic performance analysis framework upon addressing relevant factors that affect the performance of a voice application within a heterogeneous network environment is presented [16]. An algorithm is also introduced that takes into account the real-time packet error pattern along with RSS.

A dynamic decision model (QAVHMN) to make the accurate vertical handoff decisions by determining the best network based on, dynamic factors such as RSS of network and Signal-to-Noise Ratio (SNR), Link capacity(offered bandwidth) and power consumption[17]. This model not only meets the individual user needs but also improves the whole system performance by reducing the unnecessary handoffs.

In [11], the different RSS threshold values for handoff is found depending on factors like the velocity of the MT, the latency of the handoff process the type of network the MT is presently in and the type of network with which the MT is trying to initiate handoff and the size of the CN/WLAN/HIPERLAN cell the MT is presently residing. It ensures least amount of handoff failure probability, thus providing sufficient QoS for delay sensitive and real time services

A location aided Hierarchical Mobile IPv6 (HMIPv6) architecture for vertical handoff in 4G networks is designed [18]. For predicting the mobility of the nodes, a route prediction technique is presented. After predicting the mobility, AP selection is done by the route prediction server considering load and round trip time as decision parameters. In this technique, at frequent time intervals, mobile node transmits received signal strength indicator (RSSI) of adjacent access points (AP) to a Route Prediction Server (RPS). Based on RSSI threshold, RPS identifies the location of mobile nodes.

The Sugeno Fuzzy Inference system is used to find the decision for vertical handover [6]. The inference use the crisp input values for network parameters such as available bandwidth, network load and signal strength. The value of these network parameters are generated by event generator and are feed fuzzy inference system. The output of the fuzzy system is handover decision.

A handoff decision process based on RSS, MN speed and Network traffic that offers seamless vertical handoff to end users across 4G wireless networks [13]. Modifying the Client side Mobile IP is illustrated and with this modification the handoff system is simple, scalable and cost effective. The handoff system influences the packet drop rate in tight and loose integration approach under different load conditions.

An algorithm for handoff decision when MN need to switch to other network due to poor network service [7], a handoff decision is done based on both RSS and available bandwidth detail which is collected in database. The new scheme is compared with existing RSS based handoff scheme and it gives better performance in terms of PDR, total number of handoff, total time taken for handoff, total packet loss and channel utilization.

A novel vertical handoff decision algorithm for overlay wireless networks (NFLVHADPM) consisting of cellular and wireless local area networks (WLANs) is designed [20]. The target network is selected using a fuzzy logic-based normalized quantitative decision algorithm which, in addition to usual parameters such as the current RSS and the available bandwidth, also takes a prediction of the RSS into account, resulting in a more accurate handoff.

A vertical handover algorithm for vehicular communication [15], uses (RSS), initial delay for connection establishment, network traffic load and bandwidth offered, service context like usage cost, user contexts like type of application and terminal context like speed of the vehicle as different attributes of this algorithm. RSS is considered as the triggering factor, i.e, a network will be considered as an alternative only if its RSS is above threshold.

\subsection{Power Consumption}

The wireless devices running on battery need to limit the power consumption. If the battery level decreases, switching for a network to another network with low power consumption can provide a longer usage time. The power requirement becomes a critical issue especially if the hand held battery is low. In such situations, it is preferably transferred to an attachment point, and this will extend battery life. The attachment to the closest AP or BS is known to consume the least power for individual mobile devices at a given instant. So if battery level is low the MT must handoff to the closest AP or BS provided RSS is above threshold. The number of users also increases the congestion and in turn even the nearest AP or BS consumes more power.

A dynamic decision model to make the right vertical handoff decisions is developed and this not only meets the individual needs but also improves the whole system performance by reducing the unnecessary handoff [17]. A service history-based VHO algorithm is introduced to reduce unnecessary handoffs and call dropping probability in addition to QoS parameter considerations. Simulation results show that the proposed VHO algorithm outperforms existing algorithms.

A VHO decision algorithm is developed that enables a wireless access network to not only balance the overall load among all attachment points (e.g., base stations and access points) but also maximize the collective battery lifetime of Mobile Nodes [21]. In addition, when ad hoc mode is applied to 3/4G wireless data networks, 
VANETs, and IEEE 802.11 WLANs for a more seamless integration of heterogeneous wireless networks, a route-selection algorithm is devised for forwarding data packets to the most appropriate attachment point to maximize collective battery lifetime and maintain load balancing.

SeaMo, a vertical handoff (VHIHWN) that considers various parameters like RSSI, link quality metric, end-to-end available bandwidth, battery power, network usage costs etc., in making the VHO decision is presented [19]. It has been tested in a realistic scenario with a 3G access network and multiple WLAN segments. It has performed satisfactorily and handed off between $3 \mathrm{G}$ and WLAN. The operational usage of $3 \mathrm{G}$ uses higher power than that for WLAN, the idle battery usage of WLAN is higher than that of 3G. Including available battery power as a VHO decision parameter enables a faster handoff to WLAN, from $3 \mathrm{G}$ and delays the handoff from $3 \mathrm{G}$ to WLAN.

A simple two step robust vertical handoff decision algorithm ( SRVHAHWMN) is presented [22]. The first step describes the quick evaluation method for the pre-handoff decision. The second step presents the handoff decision function for handoff execution. For resource-poor mobile nodes, vertical handoff decision procedure only considers the first step. On the other hand, for resource-rich mobile nodes, vertical handoff procedure would consider both steps. This approach can reduce energy consumption on mobile nodes, especially on low energy mobile nodes.

\subsection{Throughput}

Network throughput refers to the average data rate of successful data or message delivery over a specific communications link. Network throughput is measured in bits per second (bps). Maximum network throughput equals the TCP window size divided by the round-trip time of communications data packets. As network throughput is considered in dynamic metrics for making decision of $\mathrm{VHO}$, it is one the important requirement to be considered for the VHO.

A multi criteria vertical handoff decision algorithm ( MNHGAHWN) is presented which will select the best available network with optimized parameter values [23]. In their model, they have taken into consideration the following network parameters for vertical handoff decision function (i)bandwidth(B) (ii)latency(L) (iii)SNR (iv)throughput(TH) (v)cost(C) (vi)power consumption(P) and the network with minimum latency, cost, SNR and power consumption and maximum throughput will be selected, so that an appropriate QoS level can be maintained and the number of handoff can be minimized for all the networks .

In [24], a decision function (EDHMHN) is presented in which the system considers all the available network and user parameters (e.g., host velocity, battery status, Wi-Fi AP's current load, and WiMAX BS QoS guarantees), and performs technology selection such that an overall system performance metric is optimized. It defines a new system-wise entity that is activated when a user is in an area with overlapping access technologies and needs to decide the best technology to be used, where the entity performs technology selection in order to optimize the overall system performance metric in terms of throughput and capacity limitation.

\subsection{Network Load}

Network load is to be considered during effective handoff. It is important to balance the network load to avoid deterioration in quality of services. Variations in the traffic loads among cells will reduce the trafficcarrying capacity. To provide a high quality communication service for mobile subscribers and to enhance a high traffic-carrying capacity when there are variations in traffic, network load must be paid attention.

A model is developed that analyzes the case in which a vehicle is kept at different positions of a highway, and the network ranking optimizes the "best" network in multiple constraint environments [15]. This evaluation technique requires knowledge of vehicular speed, RSS, type of application (bandwidth requirements), network traffic load, usage cost of service and initial delay for connection establishment.

The proposed methodology avoids unnecessary handovers and hence encounters "ping-pong effect".

SeaMo[19], a vertical handoff that considers various parameters like RSSI, link quality metric, end-toend available bandwidth, battery power, network usage costs in making the VHO decision. It has been tested in a realistic scenario with a $3 \mathrm{G}$ access network and multiple WLAN.

A novel vertical handoff decision algorithm for overlay wireless networks consisting of cellular and WLANs is given [20]. It has performed satisfactorily and handed off between $3 \mathrm{G}$ and WLAN. To reduce system load, a pre-decision method is employed before actual handoff decision to filter out users with high mobility or low RSS from using the WLAN. Simulation results show that the given algorithm can reduce the call-dropping probability as well as unnecessary handoffs in heterogeneous network environments.

In [11], a scheme in which the handoff decision will depend on the type of network the MT is presently in and also the type of network it is attempting handoff to ensure least amount of handoff failure probability, thus providing sufficient QoS for delay sensitive and real time services is presented. Effective handoff schemes also ensure minimal false handoff initiation probability, which leads to congestion and hence dropping of calls. 


\subsection{User Preferences}

When handover happens, the users have more options for heterogeneous networks according to their preferences and network performance parameters. The user preferences could be preferred networks, user application requirements (real time, non-real time), service types (Voice, data, video), QoS (It is a set of technologies for managing network traffic in a cost effective manner to enhance user experiences for wireless environments) etc. User Preferences can also be considered for VHO in next generation wireless networks.

A novel handover algorithm is presented which uses AHP to solve decision problem in multiple constraint environment and considers user preference in the algorithm [15]. The method decides the priority of radio access network that is most suitable for user's application at a particular vehicular speed in the constraint resource environment. Sensitivity analysis is done to justify the design of the algorithm. It is well suited for network selection in Intelligent Transportation Systems (ITS).

A vertical handoff scheme is presented in [19] which makes use of fuzzy logic approach to make decisions. The decision attributes are the processed parameters obtained from Pre Handoff (PHE) module. They are cost, user preference for the access network and battery power consumed by the access technology.

\subsection{Cost}

A multi criteria algorithm for handoff should also consider the network cost factor. The cost is to be minimized during VHO in wireless networks. The new call arrival rates and handoff call arrival rates can be analyzed using cost function. Next Generation heterogeneous networks can combine their respective advantages on coverage and data rates, offering a high QoS to mobile users. In such environment, multi-interface terminals should seamlessly switch from one network to another in order to obtain improved performance or at least to maintain a continuous wireless connection. Therefore, network selection cost is important in handoff decisions.

A novel handover algorithm presented uses AHP and in the first level of AHP, "best network" selection is made for seamless vehicular communication [15]. In order to reach this goal four criteria have been defined: usage cost, speed of the vehicle (mobility), type of application (bandwidth), network traffic load and initial delay to setup the communication link. If QoS is equivalent for two networks then user will always opt for a network which offers low cost of service.

The vertical handoff scheme designed considers various parameters like RSSI, link quality metric, endto-end available bandwidth, battery power, network usage costs in making the VHO decision [19]. In Handoff Decision Making \& Trigger (HDT) module, a fuzzy logic approach is used to make a decision. The decision attributes are the processed parameters obtained from PHE module. They are cost, user preference for the access network and battery power consumed by the access technology.

A multi criteria vertical handoff decision algorithm presented which will select the best available network with minimum latency, cost, SNR and power consumption and maximum throughput, so that an appropriate QoS level can be maintained and the number of handoff can be minimized for all the networks [23].

\begin{tabular}{|l|l|}
\hline \multicolumn{1}{|c|}{ Re-searchers } & \multicolumn{1}{|c|}{ Title of Handoff Mechanism } \\
\hline Pramod Goyal, and S. K. Saxena & $\begin{array}{l}\text { A Dynamic Decision Model for Vertical Handoffs across } \\
\text { Heterogeneous Wireless Networks (DDMVH) }\end{array}$ \\
\hline $\begin{array}{l}\text { Sourav Dhar, Amitava Ray, } \\
\text { Rabindranath Bera }\end{array}$ & $\begin{array}{l}\text { Design and Simulation of Vertical Handover Algorithm for Vehicular } \\
\text { Communication (DSVHAVC) }\end{array}$ \\
\hline $\begin{array}{l}\text { Seema K, Gopi Krishna S Garge, } \\
\text { Anand S V R, \& Malati Hegde }\end{array}$ & $\begin{array}{l}\text { A Vertical Handoff Implementation for Heterogeneous Wireless } \\
\text { Networks (VHIHWN) }\end{array}$ \\
\hline $\begin{array}{l}\text { Liu Xia, Ling-ge Jiang and Chen } \\
\text { He }\end{array}$ & $\begin{array}{l}\text { A Novel Fuzzy Logic Vertical Handoff Algorithm with Aid of } \\
\text { Differential Prediction and Pre-Decision Method ( NFLVHADPM) }\end{array}$ \\
\hline $\begin{array}{l}\text { Debabrata Sarddar, Shovan Maity, } \\
\text { Arnab Raha, Ramesh Jana, Utpal } \\
\text { Biswas, M.K. Naskar }\end{array}$ & $\begin{array}{l}\text { A RSS Based Adaptive Hand-Off Management Scheme In } \\
\text { Heterogeneous Networks (RBAHMS) }\end{array}$ \\
\hline $\begin{array}{l}\text { Jayalakshmi.S, Kumari Khushboo, } \\
\text { Kirubhashri G.M. \& M. L. Alphin } \\
\text { Ezhil Manuel }\end{array}$ & \multicolumn{1}{|c|}{ A QoS Aware Vertical Handover In Mobile Network(QAVHMN) } \\
\hline $\begin{array}{l}\text { Mrs.Chandralekha , Dr.Praffula } \\
\text { Kumar Behera }\end{array}$ & $\begin{array}{l}\text { Minimization of Number of Handoff using Genetic Algorithm in } \\
\text { Heterogeneous Wireless Networks ( MNHGAHWN) }\end{array}$ \\
\hline $\begin{array}{l}\text { A. Dvir, R. Giladi, I. Kitroser, M. } \\
\text { Segal }\end{array}$ & $\begin{array}{l}\text { Efficient Decision Handoff Mechanism for Heterogeneous Networks } \\
\text { EDHMHN) }\end{array}$ \\
\hline $\begin{array}{l}\text { Wonjun Lee, Eunkyo Kim, Inkyu } \\
\text { Lee, Choonhwa Lee et.al }\end{array}$ & $\begin{array}{l}\text { Movement-Aware Vertical Handoff of WLAN and Mobile WiMAX for } \\
\text { Seamless Ubiquitous Access (MAVHWWi) }\end{array}$ \\
\hline $\begin{array}{l}\text { Daojing He , Caixia Chi , Sammy } \\
\text { Chan Chun Chen, Jiajun Bu, } \\
\text { Mingjian Yin }\end{array}$ & $\begin{array}{l}\text { A Simple and Robust Vertical Handoff Algorithm for Heterogeneous } \\
\text { Wireless Mobile Networks ( SRVHAHWMN) }\end{array}$ \\
\hline
\end{tabular}

Table 1: Title of the existing handoff mechanisms with respect to Researchers 


\begin{tabular}{|c|c|c|c|c|c|c|c|c|}
\hline \multirow[b]{2}{*}{ Handoff mechanism } & \multicolumn{8}{|c|}{ Parameter criteria } \\
\hline & RSS & Bandwidth & Power & Speed & $\begin{array}{l}\text { User } \\
\text { Preference }\end{array}$ & $\begin{array}{l}\text { Network } \\
\text { load }\end{array}$ & Cost & Throughput \\
\hline DDMVH [10] & $\checkmark$ & $\downarrow$ & 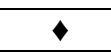 & $\diamond$ & & & & \\
\hline DSVHAVC [15] & $\downarrow$ & & & $\downarrow$ & $\downarrow$ & 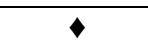 & $\downarrow$ & \\
\hline VHIHWN [19] & $\checkmark$ & $\downarrow$ & $\downarrow$ & & $\downarrow$ & 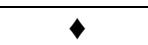 & $\downarrow$ & \\
\hline NFLVHADPM [20] & $\downarrow$ & $\downarrow$ & $\bullet$ & & & $\downarrow$ & & \\
\hline RBAHMS [11] & $\bullet$ & & & 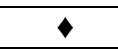 & & 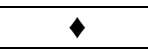 & & \\
\hline QAVHMN [17] & $\checkmark$ & $\downarrow$ & 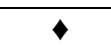 & & & & & \\
\hline MNHGAHWN [23] & & $\downarrow$ & $\checkmark$ & & & & $\downarrow$ & $\checkmark$ \\
\hline EDHMHN [24] & $\bullet$ & $\bullet$ & 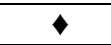 & $\bullet$ & & & & $\bullet$ \\
\hline MAVHWWi [14] & $\downarrow$ & & & $\downarrow$ & & & & 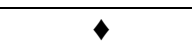 \\
\hline SRVHAHWMN [22] & $\downarrow$ & $\downarrow$ & $\downarrow$ & $\downarrow$ & & & $\downarrow$ & \\
\hline
\end{tabular}

Table 2: Parameters used in the existing handoff mechanisms

\section{Conclusion}

Seamless vertical handoff is still a challenging issue due to switching of mobile nodes amongst next generation heterogeneous networks. In this paper, we have highlighted some of the existing vertical handoff mechanisms and decision algorithms in the literature. The next generation heterogeneous wireless network has created still new challenges. In this paper, the important parameters for vertical handoff are discussed and some of them are Bandwidth, velocity, RSS, speed, throughput, Network load, and cost and user preferences. Selection of parameters for designing a vertical handoff mechanism is very crucial for the development of vertical handoff mechanism in next generation heterogeneous wireless networks. In this paper the evaluation of the existing vertical handoff mechanisms are done with respect to the parameters discussed. The evaluation indicates the necessity to design a VHO mechanism for next generation heterogeneous wireless network that satisfy multiple parameters. It is very difficult to select all the parameters during the decision of VHO mechanism, but the main parameters of the existing work are bandwidth, signal strength, and the decision model of VHO mechanism considering these parameters may yield better results.

\section{References}

[1] E. Arun \& R.S Moni, (June 2010) “A Novel Decision Scheme for Vertical Handoff in 4G Wireless Networks”, GJCST Computing Classification, Page 28 Vol.10 Issue 5 Ver.1.0

[2] Ahmed H. Zahran and Ben Liang, (Feb 2010) "Performance Evaluation Framework for Vertical Handoff Algorithms in Heterogeneous Networks".

[3] Sung H. Kim and Yeong M. Jang, "Soft Qos-Based Vertical Handover Scheme for WLAN and WCDMA Networks using Dynamic Programming Approach".

[4] Dong Ma \& Maode Ma,(April 2012) "A QoS Oriented Vertical Handoff Scheme for WiMAX/WLAN Overlay Networks", IEEE Transactions on Parallel and Distributed Systems, Vol 23, No 4.

[5] K. Radhika et al,( Oct 2011),"AHP and Group Decision Making for Access Network Selection in Multi-Homed Mobile Terminals", IJCSE, Vol. 3 No. 10.

[6] Manoj Sharma \& Dr. R.K.Khola, “An Intelligent Approach for Handover Decision in Heterogeneous Wireless Environment", International Journal of Engineering (IJE), Volume (4): Issue (5).

[7] Mithun B Patil,( Oct 2011) "Vertical Handoff in Future Heterogenous 4G Network", IJCSNS International Journal of Computer Science and Network Security, VOL.11 No.10.

[8] Mohammad Rafiq, Seema Kumar, Nagaraj Kammar, Guru Prasad,Gopi Krishna S Garge, Anand SVR, Malati Hegde, “A Vertical Handoff Decision Scheme for End-to-End QoS in Heterogeneous Networks: An Implementation on a Mobile IP Testbed”.

[9] Presila Israt, Namvi Chakma, and M. M. A. Hashem, (Dec 2009)" A Fuzzy Logic-Based Adaptive Handoff Management Protocol for Next-Generation Wireless Systems", Journal of Networks, Vol.4, No.10.

[10] Pramod Goyal, and S. K. Saxena, "A Dynamic Decision Model for Vertical Handoffs across Heterogeneous Wireless Networks“. World Academy of Science, Engineering and Technology 412008.

[11] Debabrata Sarddar, Shovan Maity, Arnab Raha, Ramesh Jana, Utpal Biswas, M.K. Naskar, ”A RSS Based Adaptive Hand-Off Management Scheme In Heterogeneous Networks", IJCSI International Journal of Computer Science Issues, Vol. 7, Issue 6, November 2010.

[12] Debabrata Sarddar, Tapas Jana, Souvik Kumar Saha, Joydeep Banerjee ,Utpal Biswas, M.K. Naskar, "Minimization of Handoff Failure Probability forNext-Generation Wireless Systems “,International Journal of Next-Generation Networks (IJNGN) Vol.2, No.2, June 2010.

[13] S.V.Saboji, C.B.Akki," A Client Based Vertical Handoff System in 4G Wireless Networks", Journal of Advances in Information Technology, Vol 1, No.4, Nov 2010.

[14] Wonjun Lee, Eunkyo Kim, Joongheon Kim, Inkyu Lee, Choonhwa Lee, "Movement-Aware Vertical Handoff of WLAN and Mobile WiMAX for Seamless Ubiquitous Access", IEEE Transactions on Consumer Electronics, Vol. 53, No. 4, NOVEMBER 2007.

[15] Sourav Dhar, Amitava Ray, Rabindranath Bera," Design and Simulation of Vertical Handover algorithm for Vehicular Communications", International Journal of Engineering Science and Technology Vol. 2(10), 2010, 5509-5525. 
[16] Tariq M. Ali, Mohammad Saquib, and Chaitali Sengupta ," Performance Analysis Framework and Vertical Handover Triggering Algorithms for Voice over WLAN/Cellular Network"

[17] Jayalakshmi.S , Kumari Khushboo, Kirubhashri G.M. \& M. L. Alphin Ezhil Manuel,’(2012) A QoS Aware Vertical Handover In Mobile Network", International Journal of Communication Network and Security (-1882 Volume IJCNS) ISSN: 2231. 1 , Issue. 3

[18] Shiva Prasad Kaleru, Damodaram Avula," Location Aided HMIPv6 Architecture for Vertical Handoff in 4G Networks", American Journal of Scientific Research ISSN 1450-223X Issue 40(2011), pp. 32-45.

[19] Seema K, Gopi Krishna S Garge, Anand S V R, and Malati Hegde,"Experiences with SeaMo: A Vertical Handoff Implementation for Heterogeneous Wireless Networks" Proceedings of the Asia-Pacific Advanced Network 2011 v. 32, p. 79-90.

[20] Liu Xia, Ling-ge Jiang and Chen He, “A Novel Fuzzy Logic Vertical Handoff Algorithm with Aid of Differential Prediction and Pre-Decision Method”, IEEE Communications Society, ICC 2007 proceedings.

[21] SuKyoung Lee, Member, IEEE, Kotikalapudi Sriram, Fellow, IEEE, Kyungsoo Kim, Yoon Hyuk Kim, and Nada Golmie, Member, IEEE, “ Vertical Handoff Decision Algorithms for Providing Optimized Performance in Heterogeneous Wireless Networks”, IEEE Transactions on Vehicular Technology, Vol. 58, No. 2, February 2009.

[22] Daojing He , Caixia Chi, Sammy Chan ,Chun Chen, Jiajun Bu, Mingjian Yin,” A Simple and Robust Vertical Handoff Algorithm for Heterogeneous Wireless Mobile Networks", (C) Springer Science+Business Media, LLC. 2010.

[23] Mrs.Chandralekha, Dr.Praffula Kumar Behera ," Minimization of Number of handoff using Genetic Algorithm in Heterogeneous Wireless Networks", International Journal of Latest Trends in Computing (E-ISSN: 2045-5364) 24 Volume 1, Issue 2, December 2010 .

[24] A. Dvir, R. Giladi, I. Kitroser, M. Segal, “Efficient Decision Handoff Mechanism for Heterogeneous Networks ”, International Journal of Wireless and Mobile Networks (IJWMN), Vol 2, No.1, February 2010.

[25] Mandeep Kaur Gondara1 and Dr. Sanjay Kadam, "Requirements of Vertical Handoff Mechanism in 4G Wireless Networks", International Journal of Wireless \& Mobile Networks (IJWMN) Vol. 3, No. 2, April 2011.

[26] Ionut BOSOANCA, Anca VARGATU, “An Overview of Vertical Handoff Decision Algorithms in NGWNs and a new Scheme for Providing Optimized Performance in Heterogeneous Wireless Networks “,Informatica Economică vol. 15, no. 1/2011. 